“C 2017 IEEE. Personal use of this material is permitted. Permission from IEEE must be obtained for all other uses, in any current or future media, including reprinting/republishing this material for advertising or promotional purposes, creating new collective works, for resale or redistribution to servers or lists, or reuse of any copyrighted component of this work in other works." 


\section{Analysis and Minimization of Detent End Force in Linear Permanent Magnet Synchronous Machines}

\author{
H. Z. Hu, J. Zhao, X. D. Liu, and Y. G. Guo
}

\begin{abstract}
In this paper, the end forces caused by the longitude end effects in linear permanent magnet synchronous machines (LPMSMs) are analyzed and minimized. Firstly, the left/right end forces are calculated based on an analytical model and the Maxwell stress tensor, in which the optimal integration surfaces are investigated. Then based on the spectrum analysis of the left/right end forces, two different methods are adopted to minimize the fundamental and high order harmonics, respectively. The optimal length of the primary iron is obtained from the phase difference of the fundamental and a two-step iteration instead of the trial-and-error with the finite element method. Furthermore, step-skewed auxiliary irons are added to the primary end to eliminate the high order harmonics. Thirdly, to reduce the secondary end effect when the primary moves to the secondary end, a compensation method of adding mirror permanent magnet is proposed and good results are obtained. Finally, an LPMSM prototype is manufactured and experiments are conducted. The experimental results verify the theoretical study.
\end{abstract}

Index Terms-Linear permanent magnet synchronous machine, end force, analytical model, force minimization.

\section{INTRODUCTION}

L INEAR permanent magnet (PM) synchronous machines $\Lambda_{\text {(LPMSMs) have been widely used for reciprocating servo }}$ systems, industrial robotics and high-precision positioning direct-drive systems thanks to their high force density, fast dynamic response, and simple mechanical structure [1]-[4]. However, the detent force caused by the slot and end effects in LPMSMs will reduce the positioning accuracy, introduce the vibration and noise, and deteriorate the control characteristics [5]-[8]. Similar to the conventional rotary PMSMs, the slot effect can be minimized by using skewed PMs [9], [10], slot-opening shifting [11], [12], and appropriate slot/pole combination [13]. However, the end effect is more significant and difficult to eliminate, as the end force waveform is periodic over one pole pitch [5]. Actually, according to the machine type and range of motion, the end effect may be divided into two different types: 1) only the primary end effect when the primary part moves in the middle of the secondary part for the LPMSM with long stroke; 2) both the primary and the secondary end effects for the LPMSM with short stroke or when the primary part moves near the end of the secondary part for the LPMSM with long stroke. The secondary end effect is always about $2 \sim 3$ pole pitches [14].

Among the methods for reducing the influence of the primary end effect, the most effective one is to optimize the primary length to adjust the phase difference between the left and right end forces [5], [14]-[16]. In [15], the left and right end forces are expressed in the form of Fourier series, and the optimal length of the primary is theoretically deduced. However, the actual effective length, which has some difference from the theoretical value, is still obtained by using finite element method (FEM) among a range of different lengths. In [14], the optimal length is obtained from the distance between the center of north or south pole and the peak of single-end force waveform, which is calculated by FEM on the assumption that the secondary is infinitely long and the primary is semi-infinitely long. However, in order to ensure that the single-end forces are good approximation to the actual end forces, the primary should be longer than 5-7 pole pitches to avoid the mutual interaction between the two end sides and the secondary also should be long enough to avoid the secondary end effect. Hence, this method is not suitable for the LPMSM with short primary or short stroke. In [16], the method similar to that in [14] is adopted to analyze the end forces, while the difference is that the single-end force and the optimal length are obtained by a series of experiments with different lengths. Though the experimental results are more convincing, the process would be complicated and the optimal value can be easily affected by the assembling tolerance. In [5], the magnetic field is firstly predicted by FEM and the magnetic forces on both end sides are calculated by nodal force method, which is derived from the Maxwell stress tensor. The phase difference between both forces is directly determined from the plotted data and then the optimal length is obtained. However, it is well known that the calculation accuracy of the Maxwell stress method is easily influenced by the finite element (FE) mesh discretization and the integral surface should be appropriately selected, which is not concerned in [5]. Meanwhile, the force calculation with FEM is also time-consuming, as shown later. Actually, the primary length optimization cannot eliminate the detent force completely due to that the end forces are not ideally sinusoidal and have many high-order harmonics.

The other effective method for reducing the primary end effect may be adding skewed auxiliary irons, as shown in [17]. As the waveform of end forces is periodic over one pole pitch, the optimal skewed length also should be one pole pitch, that is, the primary with skewed auxiliary irons is two pole pitches longer than the original length. However, the larger skewed length will increase the axial force and reduce the reciprocating range for the LPMSM with short stroke. Meanwhile, some 
other methods have been investigated, such as shape optimization of the exterior teeth [18], current compensation [6], [19], and PM optimization [20], and each has both the advantages and disadvantages.

For the LPMSM with short stroke, the secondary end effect is also non-negligible, while only limited literature has considered this problem [21]. In [21], the shape optimization of the edge PM is adopted to reduce the secondary end effect and improve the scanning range for the arc-linear PMSM. However, the complicated shape is difficult to fabricate.

In this paper, the accurate calculation of the left/right end forces based on an analytical model and the Maxwell stress tensor is firstly researched, which is critical and necessary for minimizing the resultant force. The analytical model proposed in [22] can consider both the primary and secondary end effects, and has sufficient accuracy for the force calculation. The optimal integral surfaces for calculating the left/right end forces are obtained by investigating the force characteristics around the primary. Secondly, according to the spectrum analysis of the left/right end forces, the optimal primary length is obtained by a two-step iteration and the fundamental is almost totally eliminated. Meanwhile, the step-skewed auxiliary irons are added to eliminate the second order harmonics and reduce other high-order harmonics. Thirdly, the secondary end effect is compensated by adding a mirror PM at the end of the secondary. Finally, a prototype of slotless LPMSM is manufactured and experiments are conducted to verify the analysis.

\section{END FoRCE CALCULATION}

As the end forces are the focus in this paper, the slots in the primary are neglected. Fig. 1 shows the analytical model of slotless LPMSM proposed in [22], which is obtained by converting the actual LPMSM in Cartesian coordinate into the arc-segment PMSM in polar coordinate, and the detailed illustration can be found in [22].

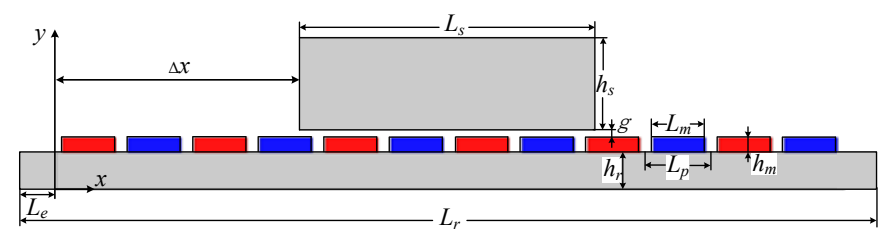

(a)

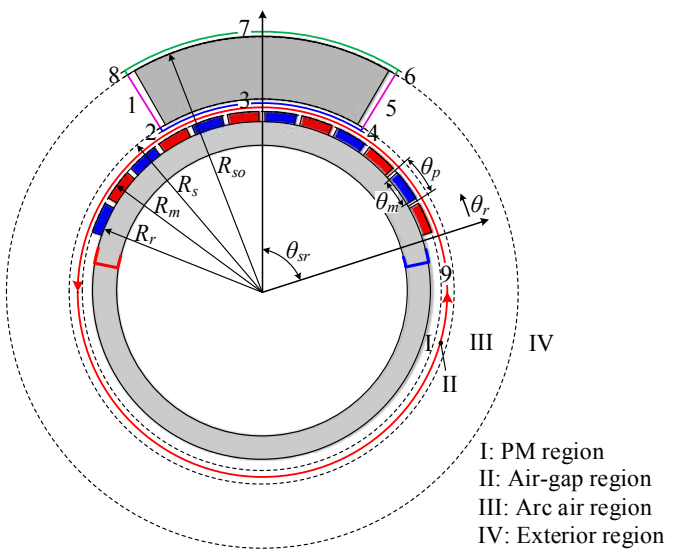

(b)
Fig. 1. The slotless LPMSM and its analytical model proposed in [22] for force calculation.

In [22], two different enclosed surfaces, that is, the surfaces $1 \sim 8$ and surface 9 , can be used to calculate the resultant force acting on the primary, as shown in Fig. 1. Though the forces obtained by the two different enclosed surfaces are equal with opposite directions, surface 9 is utilized owing to its simplicity. However, for calculating the left and right end forces separately, surface 9 is no longer suitable and some other surfaces are reselected, as shown in Fig. 2. Surface 3 and surface 7 in Fig. 1 are divided into surfaces 3-1, 3-2, 3-3, and surfaces 7-1, 7-2, 7-3, respectively. Other surfaces 1, 2, 4, 5, 6 and 8 remain the same as those in Fig. 1. The left end force is composed of the tangential force on surfaces 3-1, 7-1, and the normal force on surfaces $1,2,8$. Similarly, the right force is composed of the tangential force on surfaces 3-2, 7-2, and the normal force on surfaces $4,5,6$. The main parameters of the slotless LPMSM used for the end forces analysis are shown in Table I. The corresponding parameters of the analytical model are shown in Table II, and more details can be found in [22].

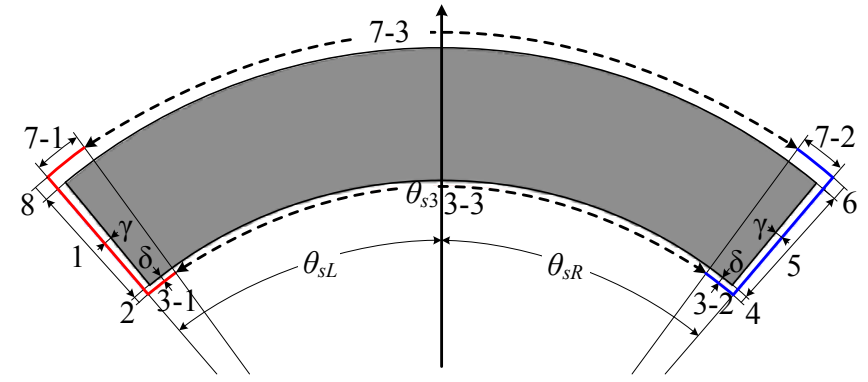

Fig. 2. Surfaces for calculating the left and right end forces.

TABLE I

PARAMETERS OF LPMSM

\begin{tabular}{|c|c|c|c|}
\hline Parameter & Value & Parameter & Value \\
\hline $\begin{array}{l}\text { Length of the primary, } L_{s} \\
\text { Height of the primary, } h_{s} \\
\text { Length of secondary iron, } L_{r} \\
\text { Length of the secondary end, } L_{e} \\
\text { Axial length, } L_{1} \\
\text { Length of air-gap, } g \\
\end{array}$ & $\begin{array}{c}50 \mathrm{~mm} \\
25 \mathrm{~mm} \\
210 \mathrm{~mm} \\
5 \mathrm{~mm} \\
50 \mathrm{~mm} \\
2 \mathrm{~mm} \\
\end{array}$ & $\begin{array}{l}\text { Number of PMs, } N_{p} \\
\text { Pole pitch, } L_{p} \\
\text { Width of PM, } L_{m} \\
\text { Thickness of PM, } h_{m} \\
\text { PM remanence, } B_{r} \\
\text { Magnetization } \\
\end{array}$ & $\begin{array}{c}20 \\
10 \mathrm{~mm} \\
10 \mathrm{~mm} \\
4 \mathrm{~mm} \\
1.27 \mathrm{~T} \\
\text { Parallel } \\
\end{array}$ \\
\hline \multicolumn{4}{|c|}{ TABLE II } \\
\hline Parameter & Value & Parameter & Value \\
\hline $\begin{array}{l}\text { Extended length, } L_{r e} \\
\text { Outer radius of PM, } R_{m} \\
\text { Span angle of the primary, } \theta_{1} \\
\text { Inner radius of the primary } R_{s} \\
\text { Outer radius of the primary } R_{s o} \\
\text { Outer radius of the secondary } \\
\text { iron, } R_{r}\end{array}$ & $\begin{array}{c}1000 \mathrm{~mm} \\
159.15 \mathrm{~mm} \\
18 \mathrm{deg} \\
161.15 \mathrm{~mm} \\
186.15 \mathrm{~mm} \\
155.15 \mathrm{~mm}\end{array}$ & $\begin{array}{l}\text { Axial length, } L_{1} \\
\text { Length of air-gap, } g \\
\text { Pole pitch, } \theta_{p} \\
\text { Span angle of PM, } \\
\theta_{m} \\
\text { PM remanence, } B_{r} \\
\text { Magnetization }\end{array}$ & $\begin{array}{c}50 \mathrm{~mm} \\
2 \mathrm{~mm} \\
3.6 \mathrm{deg} \\
3.6 \mathrm{deg} \\
1.27 \mathrm{~T} \\
\text { Radial }\end{array}$ \\
\hline
\end{tabular}

The forces on different surfaces can be obtained by the Maxwell stress sensor, respectively. For example, the normal force on surface 1 is

$$
F_{1}=\frac{L_{1}}{2 \mu_{0}} \int_{R_{s}}^{R_{s o}}\left[B_{\theta 3}^{2}\left(r, \theta_{s}\right)-B_{r 3}^{2}\left(r, \theta_{s}\right)\right] d r
$$

where $\theta_{s}=\theta_{s L}, R_{s}, R_{s o}$ are the inner and outer radii of the primary iron, respectively, and $B_{r 3}$ and $B_{\theta 3}$ are the radial and tangential flux densities in region III, respectively. The force density is 


$$
f_{1}=\frac{L_{1}}{2 \mu_{0}}\left[B_{\theta 3}^{2}\left(r, \theta_{s}\right)-B_{r 3}^{2}\left(r, \theta_{s}\right)\right] \Delta r
$$

where $\Delta r$ is the width of per unit surface.

The force density distributions on the integration surfaces at a certain position are shown in Fig. 3. It can be seen that the end forces are mainly focused on the inner corners, as marked in the circles. Meanwhile, the forces on the surfaces $6 \sim 8$ are very small and can be neglected in the following analysis. Then the left and the right end forces can be rewritten as

$$
\begin{aligned}
& F_{L}=F_{1}+F_{2}+F_{31} \\
& F_{R}=F_{32}+F_{4}+F_{5}
\end{aligned}
$$

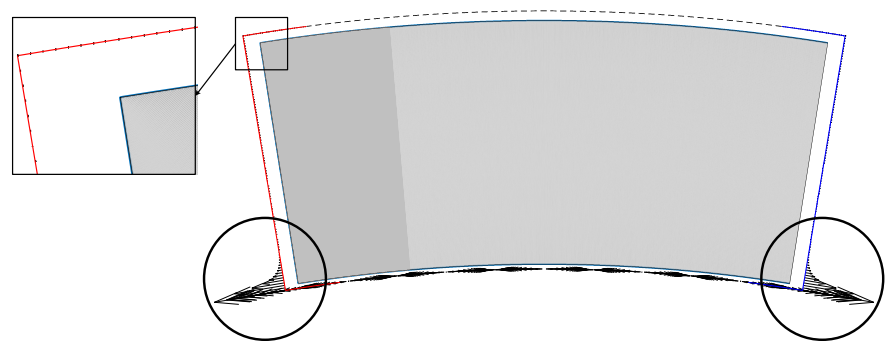

Fig. 3. Force density distributions on the integration surfaces.

It should be noted that the force densities shown in Fig. 3 may have some calculation error due to the inappropriate integration surfaces or the truncation error of the Fourier series. It is very essential to investigate the positions of the integration surfaces. From Fig. 2, it can be seen that the left/right integration surfaces are controlled by the parameters $\gamma, \delta$ and $\theta_{s 3}$. If the parameters $\gamma, \delta$ and $\theta_{s 3}$ are initially set as $0.05 \times \theta_{p}, 0.05$ $\mathrm{mm}$ and $3.0 \times \theta_{p}$, respectively, the right end forces calculated by the analytical model with different Fourier orders, such as $M=N=600,900$ and 1200, are shown in Fig. 4. Meanwhile, for the better comparison, the results obtained by FEM in which the maximum length of elements is set as $0.8 \mathrm{~mm}$ and $0.4 \mathrm{~mm}$, respectively, are also shown. As only the flux densities on the discrete points can be obtained for FEM, the numerical integration with trapezoidal integral formula is applied to calculate the end forces. It can be observed from Fig. 4 that the force waveform obtained by the FEM is not as smooth as that obtained by the analytical model, which is not conducive to the harmonic analysis, and the end force varies largely with the increase of Fourier order, which is mainly due to the truncation error and the inappropriate integration surfaces. Additionally, the computation effort of the analytical models and FEM is also shown in Table III. It can be seen that the computation time of the analytical model is much less than that of the FEM. Thus it is more suitable to optimize the integration surfaces by using the analytical model.

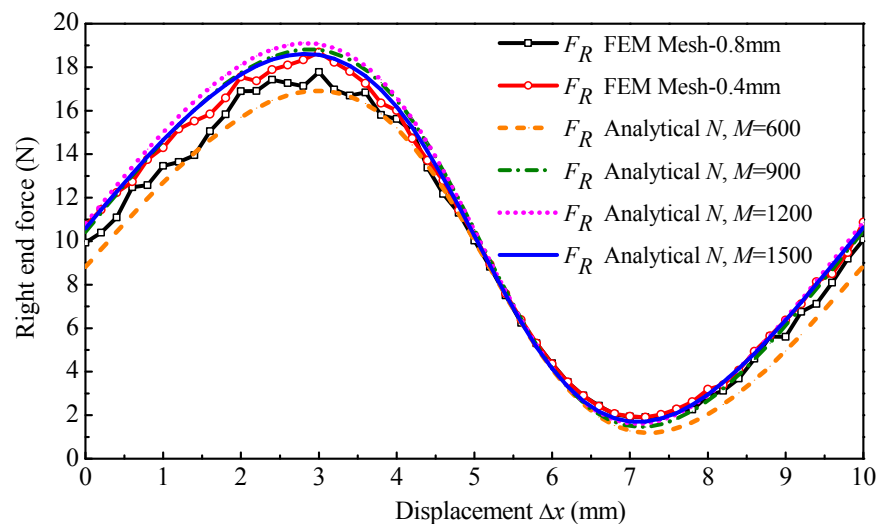

Fig. 4. The right end force obtained by FEM and analytical method.

TABLE III

COMPARISON OF COMPUTATION EFFORT BETWEEN ANALYTICAL METHOD AND FEM

\begin{tabular}{|c|c|c|c|c|c|}
\hline \multirow{2}{*}{} & \multicolumn{2}{|c|}{ FEM } & \multicolumn{3}{c|}{ Analytical model } \\
\cline { 2 - 6 } & $0.8 \mathrm{~mm}$ & $0.4 \mathrm{~mm}$ & $M, N=600$ & $M, N=900$ & $M, N=1200$ \\
\hline Time & $3 \min 7 \mathrm{~s}$ & $13 \mathrm{~min}$ & $7 \mathrm{~s}$ & $18 \mathrm{~s}$ & $34 \mathrm{~s}$ \\
\hline
\end{tabular}

A. Optimization of Parameter $\delta$ and $\theta_{s 3}$

As the forces $F_{6}, F_{7}$ and $F_{8}$ have been neglected, the resultant force $F_{t}$ obtained on surface 9 should be equal to that obtained on surfaces $1 \sim 5$, that is

$$
F_{t}=F_{L}+F_{R}+F_{33}
$$

From (5), it can be observed that $F_{L}+F_{R}$ will be equal to $F_{t}$ only if the tangential force $F_{33}$ on surface 3-3 is zero.

Before optimizing the parameters $\delta$ and $\theta_{s 3}$, the tangential force density distribution on surface 3 is firstly investigated, which is not only related to the parameter $\delta$ but also related to the relative position between the primary and the secondary. Considering the periodicity of the end forces is one pole pitch and the end forces are odd symmetric at the middle of PM, two different relative positions are investigated, as shown in Fig. 5, and the parameter $\delta$ is set as $0.01 g, 0.05 g, 0.1 g, 0.3 g$ and $0.5 g$, respectively.

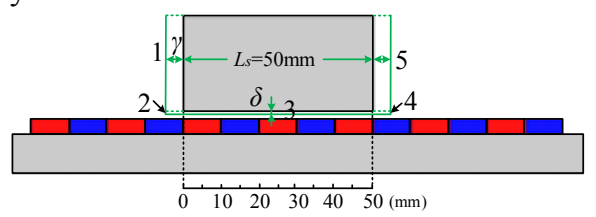

(a)

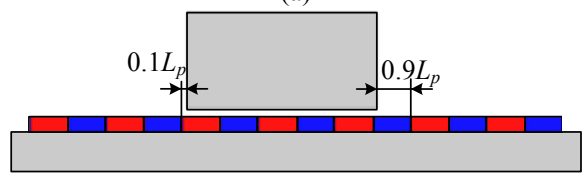

(b)

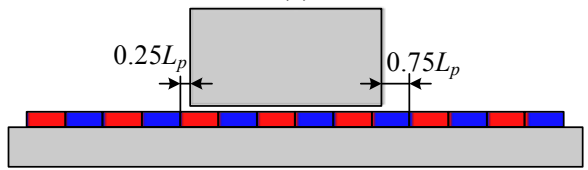

(c)

Fig. 5. Different relative positions between the primary and the secondary. (a) Position A; (b) Position B; (c) Position C.

The force density distributions on surface 3 for different relative positions are calculated and shown in Fig. 6. It can be seen that with the decrease of parameter $\delta$, the amplitude of the 
force density also decreases. This is mainly due to that when the surface 3 is close to the primary, the radial flux density is almost unchanged, while the tangential flux density decreases significantly. And in the ideal case of $\delta=0$, that is, surface 3 coincides with the inner surface of the primary, the force density should be zero due to that the boundary condition at the inner surface of the primary is $B_{\theta 2}=0$. However, this boundary condition cannot be satisfied due to the truncation error, which will lead to a high frequency fluctuations and overshoot in the tangential flux density waveform. This phenomenon is defined as Gibbs phenomenon. The waveforms of force density in Fig. 6 indirectly verify this phenomenon, e.g. the waveforms oscillate around zero when $\delta$ is small and the oscillation becomes more obvious with the decrease of $\delta$. Hence, $\delta$ should not be selected as a too small value.

It also can be observed from Fig. 6 that the force density distribution apart from the primary ends has the periodicity of one pole pitch, or to be more accurate, two pole pitches. Hence, the force $F_{33}$ will be zero if the length of the surface 3-3 $\theta_{s 3}$ is integer multiples of the pole pitch, $\theta_{s 3}=k \theta_{p}$. The forces $F_{33}$ with different $\delta$ and $\theta_{s 3}$ are shown in Table IV. It can be concluded that $F_{33}$ will be close to zero when $k$ is an even number as the period is two pole pitches. The force $F_{33}$ also varies for different relative positions, which is caused by the asymmetry of the left and the right end effects. On the other hand, the calculation error increases with the increasing of the parameter $\delta$. Hence, $\delta$ should not be selected as a too large value. The parameter $\delta$ is set as $0.05 \mathrm{~g}$ according to the compromise principle, and the parameter $\theta_{s 3}$ is set as $4 \times \theta_{p}$.

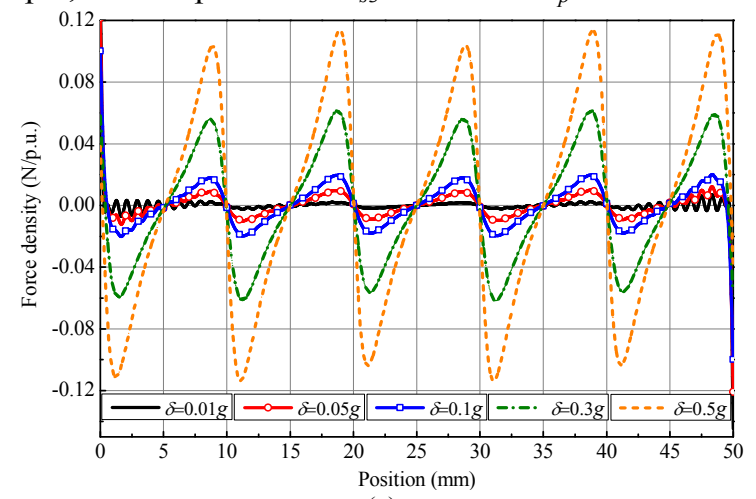

(a)

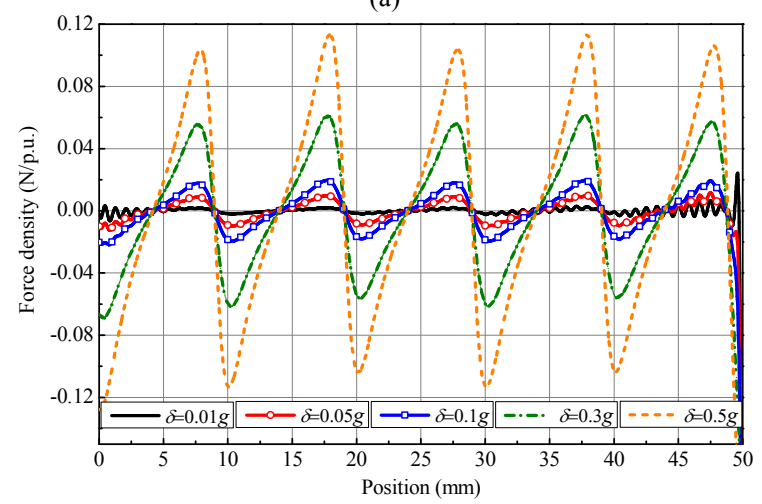

(b)

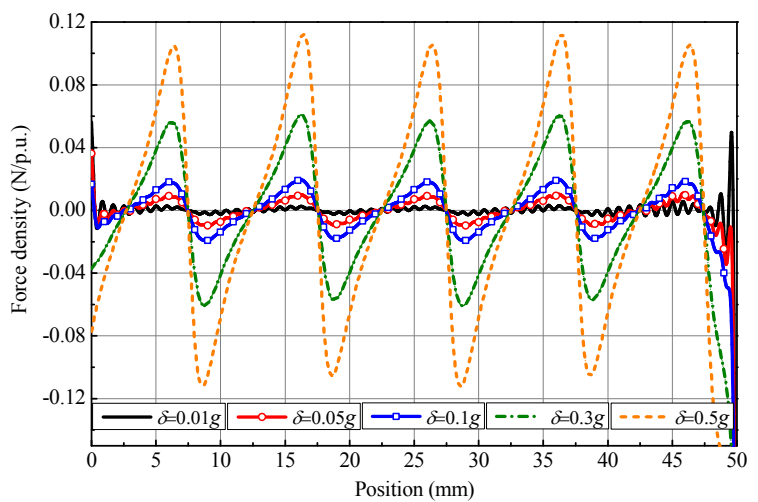

(c)

Fig. 6. Force density distributions on surface 3 for different relative positions. (a) Position A; (b) Position B; (c) Position C.

TABLE IV

FORCE $F_{33}$ WITH DifFERENT LENGTHS OF SURFACE 3-3

\begin{tabular}{|c|c|c|c|c|c|c|c|c|c|}
\hline & \multicolumn{9}{|c|}{ Tangential force (N) } \\
\cline { 2 - 11 } & \multicolumn{3}{|c|}{ Position A } & \multicolumn{3}{c|}{ Position B } & \multicolumn{3}{c|}{ Position C } \\
\hline$\theta_{\mathrm{s} 3}$ & 0.01 & 0.1 & 0.5 & 0.01 & 0.1 & 0.5 & 0.01 & 0.1 & 0.5 \\
\hline $15 \sim 35 \mathrm{~mm}$ & $\times g$ & $\times g$ & $\times g$ & $\times g$ & $\times g$ & $\times g$ & $\times g$ & $\times g$ & $\times g$ \\
$\left(2 \times \theta_{p}\right)$ & E-6 & 1.4 & 4.0 & -3.2 & 4.4 & 2.2 & -1.8 & 1.3 & 6.7 \\
\hline $10 \sim 40 \mathrm{~mm}$ & -1.8 & -7.8 & 5.7 & E-4 & E-3 & E-2 & E-5 & E-2 & E-2 \\
$\left(3 \times \theta_{p}\right)$ & E-6 & E-7 & E-6 & E-3 & 7.8 & 4.0 & 1.2 & 1.2 & 5.9 \\
\hline $5 \sim 45 \mathrm{~mm}$ & -2.0 & -1.5 & -6.9 & 3.7 & 1.5 & 7.2 & 7.3 & 3.5 & 1.7 \\
$\left(4 \times \theta_{p}\right)$ & E-5 & E-4 & E-4 & E-3 & E-2 & E-2 & E-3 & E-2 & E-1 \\
\hline
\end{tabular}

\section{B. Optimization of Parameter $\gamma$}

When the parameter $\gamma$ is optimized, the parameters $\theta_{s 3}$ and $\delta$ are assumed to remain unchanged, while the parameters $\theta_{s L}$ and $\theta_{s R}$ vary with the parameter $\gamma$. Ten different values, $0.01 \theta_{p}$, $0.03 \theta_{p}, 0.05 \theta_{p}, 0.07 \theta_{p}, 0.1 \theta_{p}, 0.2 \theta_{p}, 0.3 \theta_{p}, 0.5 \theta_{p}, 0.7 \theta_{p}$ and $1.0 \theta_{p}$, are selected for the optimization. The resultant force acting on the primary obtained by surface 9 is set as the reference value. Fig. 7 shows the resultant forces obtained by different methods. Here, $F_{L}+F_{R}$ is the total of the left and the right end forces which are obtained by (3) and (4), respectively. In order to further evaluate the degree of the force $F_{L}+F_{R}$ being close to the reference value $F_{t}$, the root mean square (RMS) error is calculated as

$$
\kappa_{e}=\sqrt{\frac{\sum_{i=1}^{N_{p t s}}\left[\left(F_{L}+F_{R}\right)_{i}-F_{t, i}\right]^{2}}{N_{p t s}}}
$$

where $N_{p t s}$ is the number of sampling points.

Fig. 8 shows the waveform of RMS error with different $\gamma$. From Fig. 7 and Fig. 8, it can be seen that with the increasing of $\gamma$, the force $F_{L}+F_{R}$ gradually becomes stable and agrees with $F_{t}$. Meanwhile the RMS error is almost reduced to zero when $\gamma \geq 0.3 \theta_{p}$. Here, $\gamma=1.0 \theta_{p}$ is selected as the optimal value. 


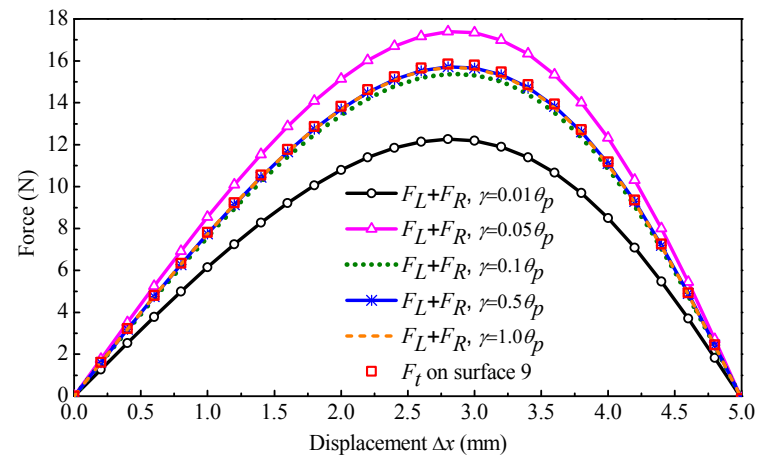

Fig. 7. Comparison on the resultant forces with different $\gamma$.

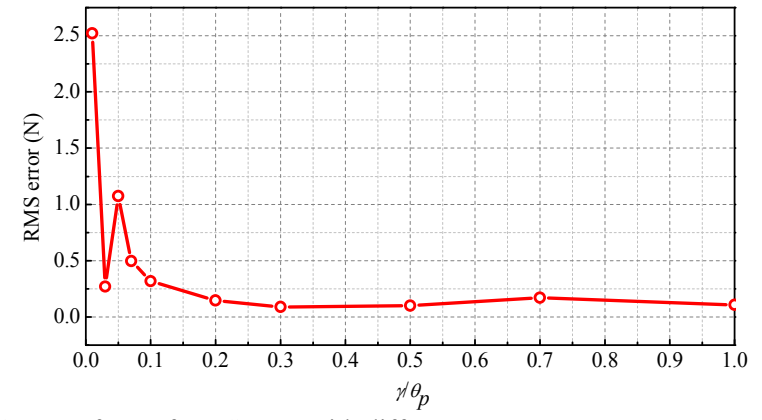

Fig. 8. Waveform of RMS error with different $\gamma$.

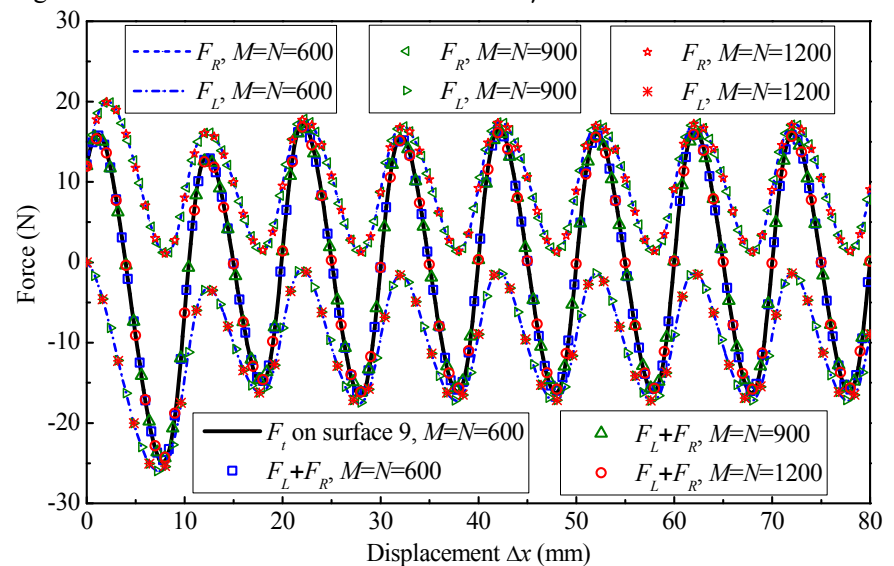

Fig. 9. End forces obtained with the optimal parameters.

With the optimal parameters $\gamma, \delta$ and $\theta_{s 3}$, the left end force, right end force and the resultant force are recalculated with different Fourier orders, as shown in Fig. 9. It can be seen that the force $F_{L}+F_{R}$ coincides with the force $F_{t}$ and they remain almost unchanged with the increasing of Fourier order. Meanwhile, the left and the right end force waveforms are no longer odd symmetrical when the primary moves to the end of the secondary.

\section{ANALYSIS AND MINIMIZATION OF THE PRIMARY END EFFECT}

In this part, the primary end effect is firstly analyzed and minimized. In order to reduce the influence of the secondary end effect, the waveforms of $F_{L}, F_{R}$ and $F_{L}+F_{R}$ during the middle operating range, e.g. $70 \mathrm{~mm} \leq \Delta x \leq 80 \mathrm{~mm}$, are employed for the further analysis, as shown in Fig. 10, and the spectrum analyses of the end forces are listed in Table V. It can be seen that the fundamental and the $2^{\text {nd }}$ harmonic are the dominant components. The amplitude of the $2^{\text {nd }}$ harmonic is about $9 \%$ of the fundamental. If the fundamental and the $2^{\text {nd }}$ harmonics are eliminated, the resultant end force will be significantly reduced. Here, the length optimization of the primary is adopted to eliminate the fundamental. However, different from the existing literature, the optimal length in this paper is obtained based on the spectrum analyses and an iteration process. To eliminate the $2^{\text {nd }}$ harmonic, the step-skewed auxiliary irons are proposed and the optimal shifted length is analyzed.

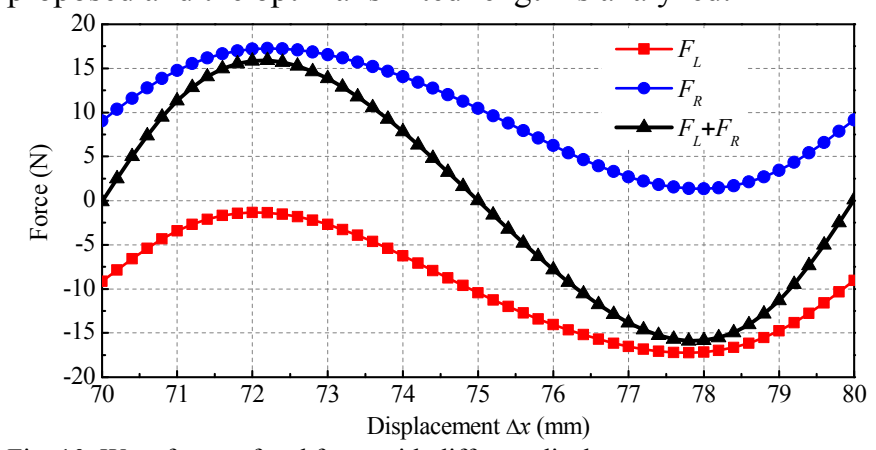

Fig. 10. Waveforms of end force with different displacements.

TABLE V

HARMONICS ANALYSES OF THE LEFT/RIGHT END FORCE

\begin{tabular}{|c|c|c|c|c|c|c|}
\hline Harmonic & & $\mathrm{DC}$ & 1 & 2 & 3 & 4 \\
\hline Left & $\begin{array}{r}\text { Amplitude } \\
\text { Phase }\end{array}$ & -9.56 & & $\begin{array}{r}0.66 \\
-97.6\end{array}$ & $\frac{0.04}{87.5}$ & $\frac{0.10}{109.7}$ \\
\hline Right & $\begin{array}{r}\text { Amplitude } \\
\text { Phase }\end{array}$ & 9.56 & $\frac{7.78}{-91.8}$ & $\frac{0.66}{-68.3}$ & $\frac{0.04}{113.7}$ & $\frac{0.10}{98.7}$ \\
\hline Resultant & $\begin{array}{r}\text { Amplitude } \\
\text { Phase }\end{array}$ & 0 & $\begin{array}{r}15.49 \\
-86.5 \\
\end{array}$ & $\frac{1.28}{-82.9}$ & $\frac{0.07}{100.6}$ & $\frac{0.20}{104.1}$ \\
\hline
\end{tabular}

A. Optimization of the primary length

According to the harmonic spectrum in Table V, the left and right end forces can be approximated as

$$
\begin{aligned}
& F_{L}(\Delta x) \approx-9.56+7.78 \cos \left(\frac{2 \pi}{L_{p}} \Delta x-\frac{81.1}{180} \pi\right)+0.66 \cos \left(\frac{4 \pi}{L_{p}} \Delta x-\frac{97.6}{180} \pi\right) \\
& F_{R}(\Delta x) \approx 9.56+7.78 \cos \left(\frac{2 \pi}{L_{p}} \Delta x-\frac{91.8}{180} \pi\right)+0.66 \cos \left(\frac{4 \pi}{L_{p}} \Delta x-\frac{68.3}{180} \pi\right)
\end{aligned}
$$

If the length of the primary is increased from $L_{s}$ to $L_{s}+\Delta L_{1}$, and the extended part is assumed to be added to the right side of the primary, as shown in Fig. 11, then the right end force can be rewritten as

$$
\begin{aligned}
F_{R}(\Delta x) \approx 9.56+7.78 \cos \left[\frac{2 \pi}{L_{p}}\left(\Delta x+\Delta L_{1}\right)-\frac{91.8}{180} \pi\right] \\
+0.66 \cos \left[\frac{4 \pi}{L_{p}}\left(\Delta x+\Delta L_{1}\right)-\frac{68.3}{180} \pi\right]
\end{aligned}
$$

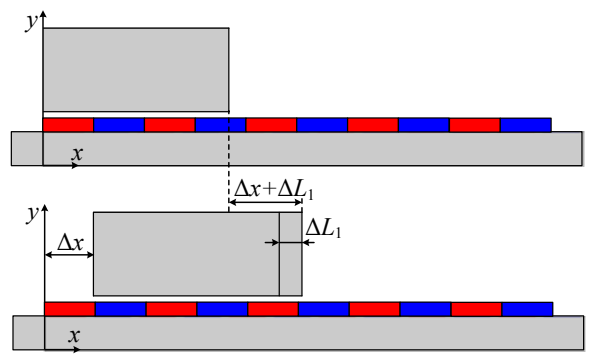

Fig. 11. Optimization of the primary length.

The fundamental can be canceled by adjusting the phase difference and the extended length $\Delta L_{1}$ should satisfy 


$$
(-81.1+91.8) \times \frac{\pi}{180}-\frac{2 \pi}{L_{p}} \Delta L_{1}=(2 k+1) \pi \quad k=0, \pm 1, \cdots
$$

where $k$ should be an integer that makes $\Delta L_{1}$ take the smallest value. Based on (10), $\Delta L_{1}$ is obtained as $5.30 \mathrm{~mm}$.

The left and right end forces are recalculated with the length $L_{s}=55.30 \mathrm{~mm}$ and shown in Fig. 12. The corresponding spectrum analyses are listed in Table VI. From Fig. 12, it can be seen that the amplitude of the resultant force is significantly reduced from $16 \mathrm{~N}$ to $2.0 \mathrm{~N}$ and the $2^{\text {nd }}$ harmonic becomes the dominant harmonic. However, with the theoretical value $L_{s}=55.30 \mathrm{~mm}$, the fundamental is not completely canceled. The amplitude of the fundamental is $0.35 \mathrm{~N}$ and the phase difference is 183.3 deg., not the optimal $180 \mathrm{deg}$., which is mainly due to that the extension of the primary right side not only affects the right end force, but also has an impact on the left end force. This illustrates that the left and right end effects interact with each other for the LPMSM with short stroke.

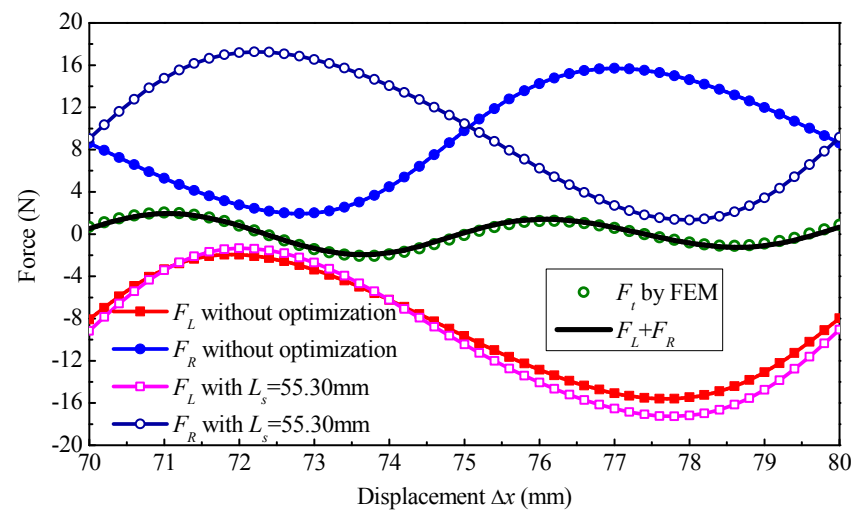

Fig. 12. Waveforms of the end forces with different lengths of the primary. TABLE VI

HARMONICS ANALYSES OF THE LEFT/RIGHT END FORCES

\begin{tabular}{|c|c|c|c|c|c|c|}
\hline Harmonics & & $\overline{\mathrm{DC}}$ & 1 & 2 & 3 & 4 \\
\hline Left & $\begin{array}{c}\text { Amplitude } \\
\text { Phase }\end{array}$ & -8.90 & $\begin{array}{r}6.74 / 81.2 \\
-8\end{array}$ & $\frac{0.72}{-81.0}$ & $\frac{0.08}{-90.7}$ & $\frac{0.04}{113.5}$ \\
\hline Right & $\begin{array}{c}\text { Amplitude } \\
\text { Phase }\end{array}$ & 8.94 & & $\frac{0.91}{-71.6}$ & $\frac{0.13}{138.3}$ & $\frac{0.05}{-53.5}$ \\
\hline Resultant & $\begin{array}{c}\text { Amplitude } \\
\text { Phase }\end{array}$ & 0.05 & & $1.62 /-75.7$ & & \\
\hline
\end{tabular}

To further reduce the fundamental, the second length optimization adopting the same process is necessary. The length of the primary is further increased from $L_{s}+\Delta L_{1}$ to $L_{s}+\Delta L_{1}+\Delta L_{2}$, and $\Delta L_{2}$ should satisfy

$$
(-81.2-95.9) \times \frac{\pi}{180}-\frac{2 \pi}{L_{p}} \Delta L_{2}=(2 k+1) \pi \quad k=0, \pm 1, \cdots
$$

Then $\Delta L_{2}$ can be obtained as $0.08 \mathrm{~mm}$. The left end force and right end force are recalculated with $L_{s}=55.38 \mathrm{~mm}$. The resultant force is shown in Fig. 13 and the spectrum analyses are listed in Table VII. It can be seen that the fundamental has been almost completely canceled with the amplitude $0.03 \mathrm{~N}$ and the phase difference $180.2 \mathrm{deg}$. The optimal length always can be obtained by two-step or three-step iteration.

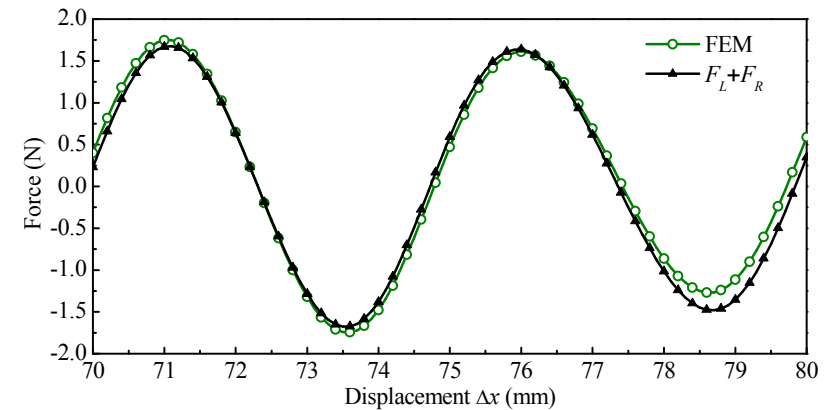

Fig. 13. Waveforms of end force after the second length optimization of the primary.

TABLE VII

\begin{tabular}{|c|c|c|c|c|c|c|}
\hline Harmonic & & $\overline{\mathrm{DC}}$ & 1 & 2 & 3 & 4 \\
\hline Left & $\begin{array}{r}\text { Amplitude } \\
\text { Phase }\end{array}$ & & $\begin{array}{r}6.73 \\
-81.3 \\
\end{array}$ & $\frac{0.71}{-81.1}$ & $\begin{array}{r}0.07 \\
-89.4\end{array}$ & $\begin{array}{l}0.04 \\
113.5\end{array}$ \\
\hline Right & $\begin{array}{r}\text { Amplitude } \\
\text { Phase }\end{array}$ & & $\frac{6.73}{98.9}$ & $\frac{0.90}{-66.2}$ & $\begin{array}{r}0.14 \\
147.8\end{array}$ & -47.5 \\
\hline Resultant & $\begin{array}{r}\text { Amplitude } \\
\text { Phase }\end{array}$ & & & & $0.12 / 179.9$ & $\begin{array}{r}0.02 / 12.5 \\
\end{array}$ \\
\hline
\end{tabular}

HARMONICS ANALYSES OF THE LEFT/RIGHT END FORCES

\section{B. Step-skewed auxiliary irons}

After the length optimization of the primary, the fundamental has been eliminated. To further eliminate the $2^{\text {nd }}$ harmonic and reduce other higher-order harmonics, step-skewed auxiliary irons are applied to the primary iron, as shown in Fig. 14.

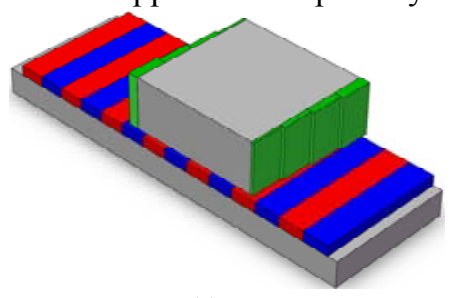

(a)

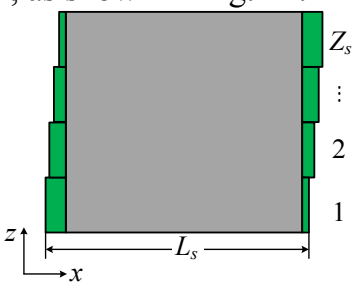

(b)
Fig. 14. Structure of step-skewed auxiliary iron. (a) 3D view; (b) 2D view.

If the auxiliary irons have $Z_{s}$ slices in the $z$ direction and the total shifted length is $L_{d}$, the shifted length of each slice is

$$
\Delta x_{i}=\frac{i-1}{Z_{s}-1} L_{d} \quad i=1,2, \cdots, Z_{s}
$$

The resultant force of the primary can be expressed by a Fourier series as

$$
F_{t}=\sum_{n=1}^{\infty} F_{t n} \cos \left(n \frac{2 \pi}{L_{p}} x+\gamma_{t n}\right)
$$

Then, the resultant force of the primary with step-skewed auxiliary irons can be

$$
\begin{aligned}
F_{t s} & =\sum_{i=1,2,3, \cdots}^{Z_{s}} \sum_{n=1}^{\infty} \frac{F_{t n}}{Z_{s}} \cos \left[n \frac{2 \pi}{L_{p}} \times\left(x+\Delta x_{i}\right)+\gamma_{t n}\right] \\
& =\sum_{n=1}^{\infty} F_{t n} G(n) \cos \left(n \frac{2 \pi}{L_{p}} x+\gamma_{t n}+\frac{n \pi L_{d}}{L_{p}}\right)
\end{aligned}
$$

where

$$
G(n)=\frac{\sin \left(Z_{s} \frac{n \pi}{Z_{s}-1} \frac{L_{d}}{L_{p}}\right)}{Z_{s} \sin \left(\frac{n \pi}{Z_{s}-1} \frac{L_{d}}{L_{p}}\right)} .
$$

The numerator of $G(n)$ is zero when the total shifted length 
$L_{d}$ is selected as

$$
L_{d}=\frac{Z_{s}-1}{Z_{s}} L_{p}
$$

Then, $G(n)$ is not zero if and only if the denominator of $G(n)$ is also zero according to the L'Hopital's rule, viz. $n=j Z_{s}, j=1,2$,

$$
\left.G\left(j Z_{s}\right)\right|_{L_{d}=\frac{Z_{s}-1}{Z_{s}} L_{p}}=\frac{\sin \left(j Z_{s} \pi\right)}{Z_{s} \sin (j \pi)}=(-1)^{j\left(Z_{s}-1\right)}
$$

That is, the $n=j Z_{s}$ harmonics cannot be eliminated. For example, when $Z_{s}=2$, the harmonics can be eliminated except the $2^{\text {nd }}, 4^{\text {th }}$, and so on; when $Z_{s}=3$, the harmonics can be eliminated except the $3^{\text {rd }}, 6^{\text {th }}$, and so on. In order to eliminate the $2^{\text {nd }}$ harmonic, $Z_{s} \geq 3$.

On the other hand, the fundamental has been eliminated by optimizing the length of the primary and the $2^{\text {nd }}$ harmonic becomes the dominant harmonic. Hence, the $2^{\text {nd }}$ harmonic should be firstly considered. If the total shifted length $L_{d}$ is selected as

$$
L_{d}=\frac{Z_{s}-1}{2 Z_{s}} L_{p}
$$

the numerator of $G(n)$ will be zero for the even components $n=2 j$, while the numerator will not be zero for the odd components $n=2 j-1$. However, the amplitude for odd components may also be reduced, as

$$
\left.G(2 j-1)\right|_{L_{d}=\frac{Z_{s}-1}{2 Z_{s}} L_{p}}=\left|\frac{1}{Z_{s} \sin \frac{(2 j-1) \pi}{2 Z_{s}}}\right|<1 .
$$

Thus, the relationship between the resultant force and the total shifted length $L_{d}$ is established. Based on the selection of $L_{d}$, the step-skewed auxiliary irons can be divided into two kinds:

$$
\text { i) } L_{d}=\frac{Z_{s}-1}{Z_{s}} L_{p}, Z_{s} \geq 3 \text {; ii) } L_{d}=\frac{Z_{s}-1}{2 Z_{s}} L_{p}, Z_{s} \geq 2 \text {. }
$$

In order to compare the effectiveness of the step-skewed auxiliary irons with different $Z_{s}$, five different cases are considered. For the first kind, two cases are considered. Case one is $L_{d}=2 L_{p} / 3$ when $Z_{s}=3$ and case two is $L_{d}=3 L_{p} / 4$ when $Z_{s}=4$. For the second kind, three cases are considered. Case three is $L_{d}=L_{p} / 4$ when $Z_{s}=2$, case four is $L_{d}=L_{p} / 3$ when $Z_{s}=3$, and case five is $L_{d}=3 L_{p} / 8$ when $Z_{s}=4$. Fig. 15 shows the waveforms of the end forces with different cases of the step-skewing, and Fig. 16 shows the comparison of the resultant end forces. It can be seen that when the displacement $\Delta x>30 \mathrm{~mm}$, the resultant end force is significantly reduced with the step-skewed auxiliary irons, and the amplitude difference among the five cases is very small. When the primary is close to the end of the secondary, the amplitudes of the resultant end force of case one and case two are slightly smaller than the other three cases. In addition, considering the manufacture complexity and the axial end force, case three is selected.

The spectrum analyses of $F_{L}+F_{R}$ during $70 \mathrm{~mm} \leq \Delta x \leq 80 \mathrm{~mm}$ of case three are listed in Table VIII. It can be seen that the $2^{\text {nd }}$ harmonic is almost completely canceled. However, the fundamental increases again, which may be due to the secondary end effect.

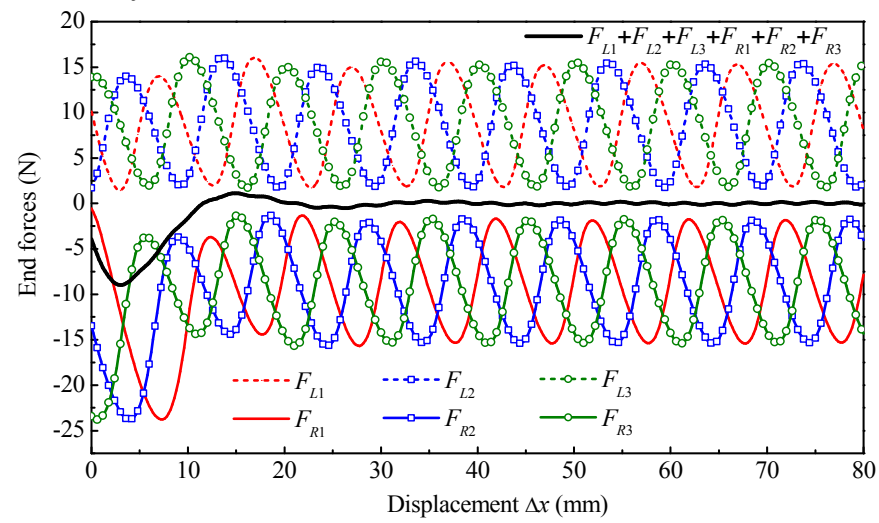

(a)

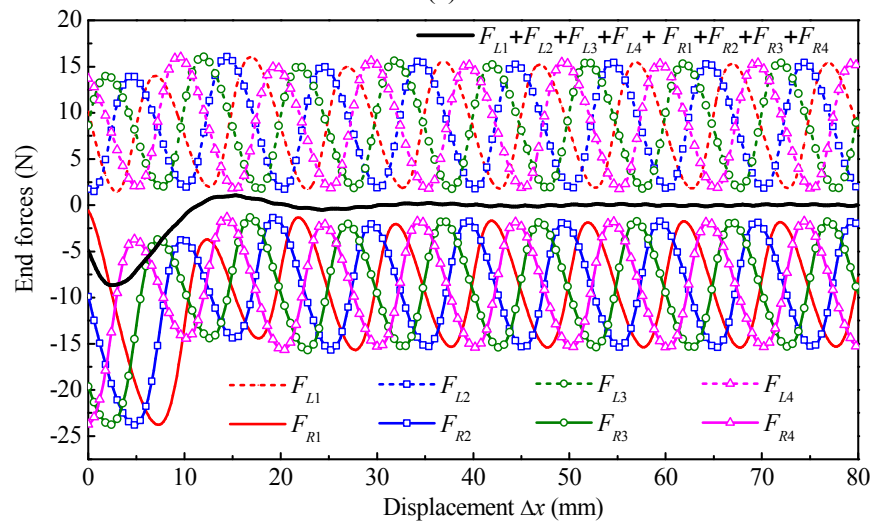

(b)

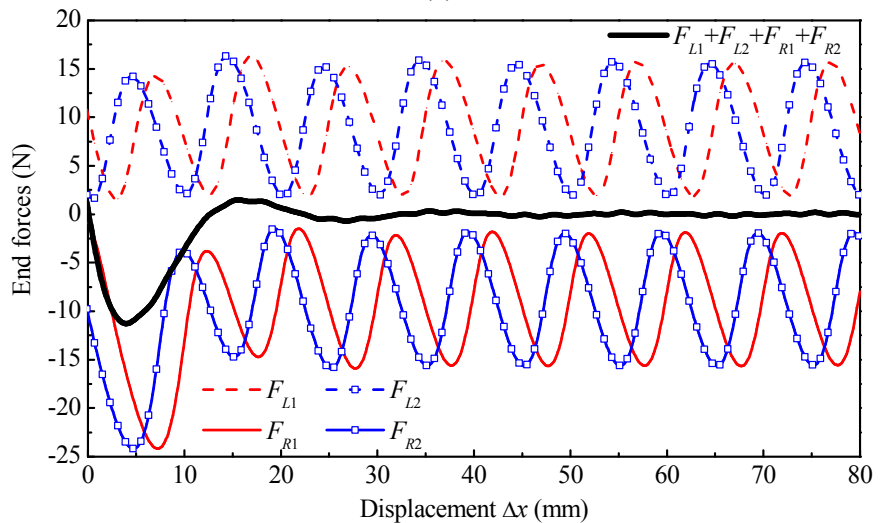

(c)

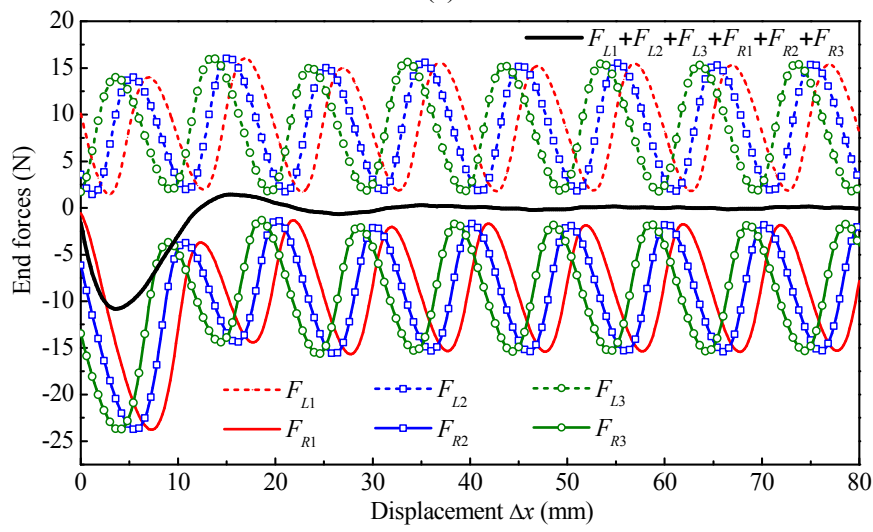

(d) 


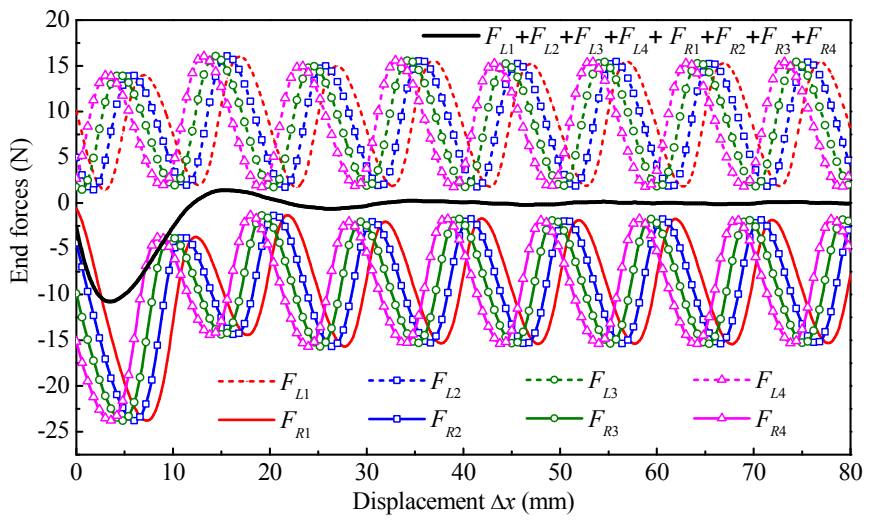

(e)

Fig. 15. Waveforms of the end forces with different cases. (a) Case one. (b) Case two. (c) Case three. (d) Case four. (e) Case five.

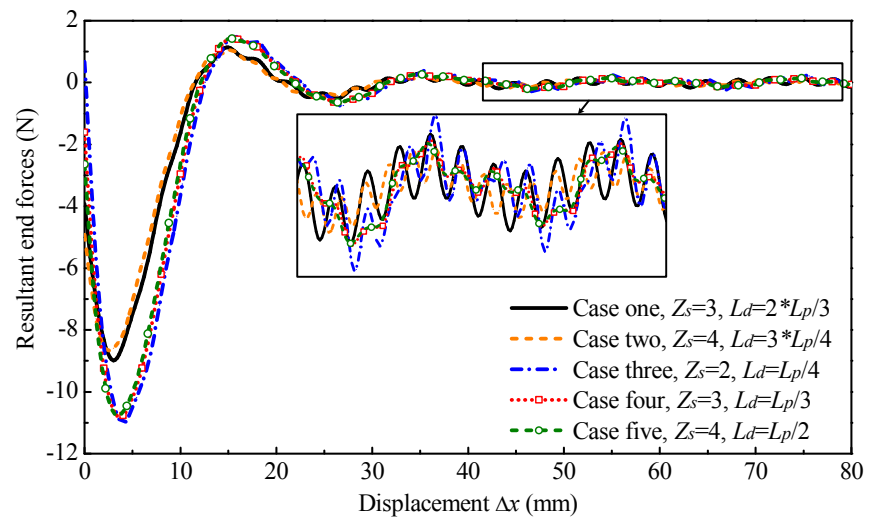

Fig. 16. Comparison of the resultant end forces with different cases.

TABLE VIII

HARMONICS ANALYSES OF THE RESULTANT END FORCES

\begin{tabular}{|c|c|c|c|c|c|c|}
\hline \multicolumn{2}{|c|}{ Harmonics } & DC & 1 & 2 & 3 & 4 \\
\hline \multirow{2}{*}{ Resultant } & \begin{tabular}{c} 
Amplitude \\
\cline { 2 - 6 }
\end{tabular} & \multirow{2}{*}{0.04} & 0.151 & 0.041 & 0.142 & 0.036 \\
& Phase & & -64.3 & -71.6 & 71.3 & -52.6 \\
\hline
\end{tabular}

\section{Compensation of the SeCondary End EFfect}

In the previous section, the resultant end force caused by the primary end effect has been minimized and in this section, the secondary end effect will be further analyzed. The forces shown in Fig. 16 indicate that when the primary is close to the end of the secondary, the amplitude and phase of the left/right forces change largely and can no more cancel each other out, and the resultant end force increases significantly. The magnetic flux distribution at the end of the secondary is shown in Fig. 17. It is clear that as the magnetic reluctance of the left part of the leftmost PM is the largest, the magnetic circuit is no longer symmetrical about the center line of the PMs, as shown in the left and right dashed boxes. Meanwhile, the asymmetry of magnetic circuit of the leftmost PM will further affect the magnetic field distribution around the second PM. The secondary end effect is about $2 \sim 3$ pole pitches.

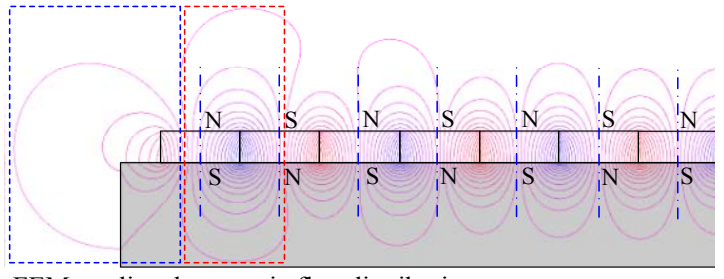

Fig. 17. FEM predicted magnetic flux distribution.

To compensate the influence caused by the secondary end effect, improving the magnetic flux distribution around the end of the secondary is very essential. In this paper, two different compensation methods are proposed and compared. The first one is to add an iron block to the left of the leftmost PM, which will reduce the magnetic reluctance of the left part of the leftmost PM, as shown in Fig. 18(a). The other is to add a mirror PM, as shown in Fig. 18(b) and the width of mirror PM is half of the normal PMs. The radial flux densities with different compensation methods are shown in Fig. 19. In Fig. 19 , the radial flux density of LPMSM with a long secondary is set as the reference value. From the comparison, it can be seen that adding an iron block can reduce the secondary end effect, while adding a mirror PM is even more effective. The magnetic field distribution with adding mirror PM is shown in Fig. 20 and it is more symmetrical than that in Fig. 17. The waveforms of the resultant end forces with different compensation methods are shown in Fig. 21. For adding mirror PM, the secondary end effect is almost completely compensated.

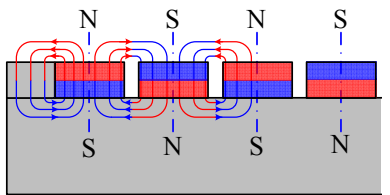

(a)

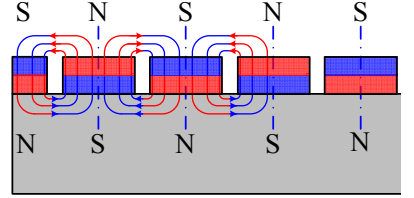

(b)
Fig. 18. Minimization of the secondary end effect. (a) Adding an iron block; (b) Adding a mirror PM.

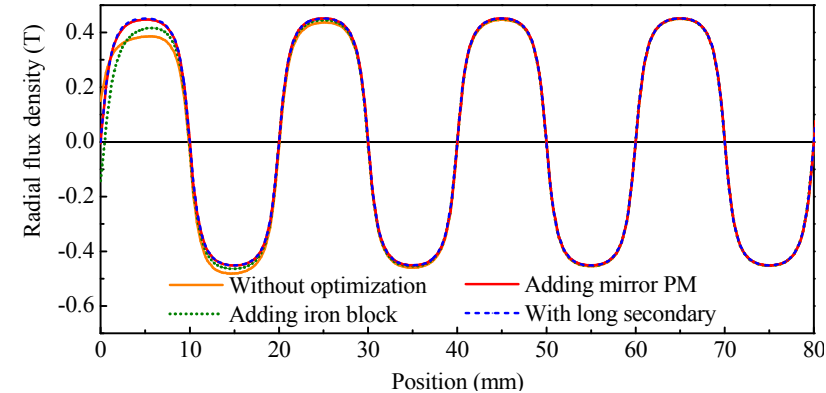

Fig. 19. Waveforms of radial flux density with different optimization methods.

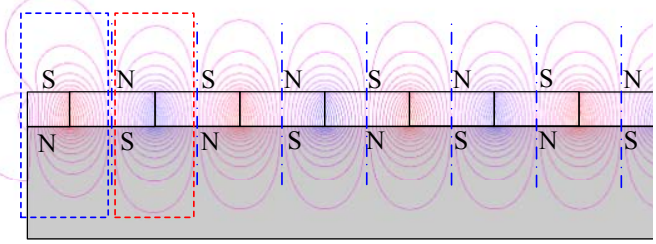

Fig. 20. Magnetic flux distribution with added mirror PM. 


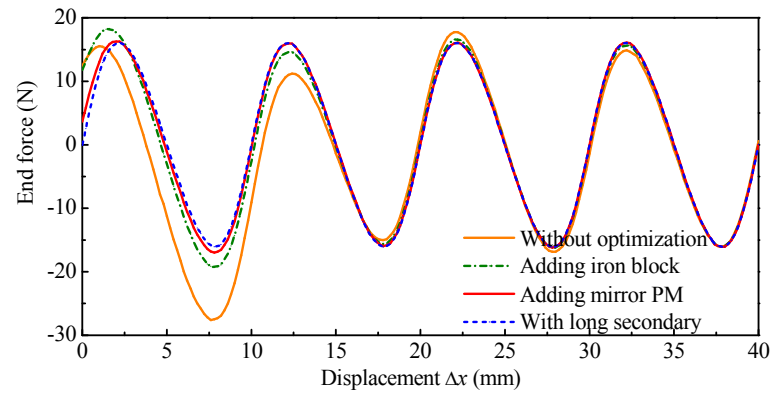

(a)

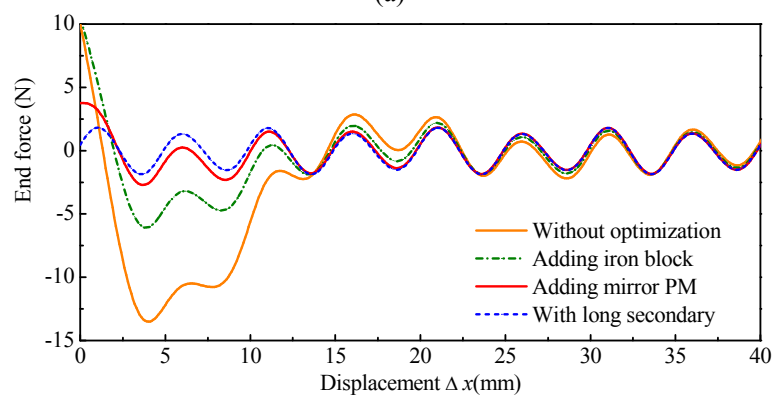

(b)

Fig. 21. Waveforms of end force with different optimization methods. (a) The primary length is $50 \mathrm{~mm}$. (b) The primary length is $55.38 \mathrm{~mm}$.

\section{VALIDATION BY EXPERIMENT}

To validate the analysis and optimization, a prototype of LPMSM is manufactured, as shown in Fig. 22. It can be seen that the primaries with the initial length $50.00 \mathrm{~mm}$, the optimal length of $55.38 \mathrm{~mm}$ and two step-skewed auxiliary irons are all fabricated. Meanwhile, the secondary with or without the mirror PM is also fabricated. To measure the end forces with different situations, an experiment platform is built, as shown in Fig. 23. In this platform, the primary and the force sensor are rigidly connected to the workbench through two bolts and a bracket, and the secondary is pushed forward step by step through another bolt. The displacement of the secondary is measured by a vernier caliper with a precision of $0.02 \mathrm{~mm}$. For the force sensor, its fullscale is $98 \mathrm{~N}$ and its precision is $0.2 \%$ of the fullscale.

The theoretical and measured values of some key dimensions in the prototype are both listed in Table IX. It can be seen that the machining errors of the initial primary length $L_{s}$ and the optimal primary length $L_{s o}$ are $0.01 \mathrm{~mm}$ and $-0.01 \mathrm{~mm}$, respectively. The actual width of each PM varies from $9.99 \mathrm{~mm}$ to $10.01 \mathrm{~mm}$, but the total length of $20 \mathrm{PMs}$ is about $200.62 \mathrm{~mm}$. It is $0.62 \mathrm{~mm}$ longer than that of the theoretical design and the pole pitch varies from $10 \mathrm{~mm}$ to $10.03 \mathrm{~mm}$, which is mainly due to the assembling error of multiple PMs. For the sake of better comparison, the pole pitch in the analytical model is revised to $10.03 \mathrm{~mm}$ and the primary lengths are revised to the measured values, while the width of each PM is still $10.00 \mathrm{~mm}$. The resultant end force waveforms obtained by analytical models and experimental tests are shown in Fig. 24(a), and the corresponding spectrum analyses of the end forces during $70 \mathrm{~mm} \leq \Delta x \leq 80 \mathrm{~mm}$ are listed in Table X. Firstly, it can be seen that the results obtained by analytical models have a good agreement with those obtained by the experiments. Secondly, the fundamental is significantly reduced from $16.86 \mathrm{~N}$ to 1.66
$\mathrm{N}$ with the length optimization of the primary. However, it is not completely eliminated, which is mainly due to that the pole pitch changes from $10.00 \mathrm{~mm}$ to $10.03 \mathrm{~mm}$ and the machining error of the primary length. Thirdly, with the step-skewed auxiliary irons, the second harmonic is reduced from $1.86 \mathrm{~N}$ to $0.13 \mathrm{~N}$. Meanwhile, the fundamental is reduced from $1.66 \mathrm{~N}$ to $0.57 \mathrm{~N}$, which can be explained based on (18). Fig. 24(b) shows the waveforms of the resultant end forces with mirror PMs at the ends of the secondary. It also can be seen that the theoretical results are consistent with the experimental ones, and the amplitude of the end forces during the end operating range is reduced from $-14 \mathrm{~N}$ to $-2.1 \mathrm{~N}$, which is slightly larger than that during the middle operating range. Through this series of comparisons, it can be concluded that the analysis and minimization of the end forces are effective, and the optimal length of the primary is sensitive to the manufacturing and assembling accuracy.

TABLE IX

THE COMPARISON OF THE THEORETICAL AND MEASUREd VALUES OF SOME KEY DIMENSIONS

\begin{tabular}{|c|c|c|}
\hline Parameters & Theoretical & Measured \\
\hline The initial length of the primary, $L_{s}$ & $50.00 \mathrm{~mm}$ & $50.01 \mathrm{~mm}$ \\
\hline The optimal length of the primary, $L_{s o}$ & $55.38 \mathrm{~mm}$ & $55.37 \mathrm{~mm}$ \\
\hline The width of the PMs, $L_{m}$ & $10.00 \mathrm{~mm}$ & $9.99-10.01 \mathrm{~mm}$ \\
\hline The total width of 20 PMs & $200.00 \mathrm{~mm}$ & $200.62 \mathrm{~mm}$ \\
\hline
\end{tabular}

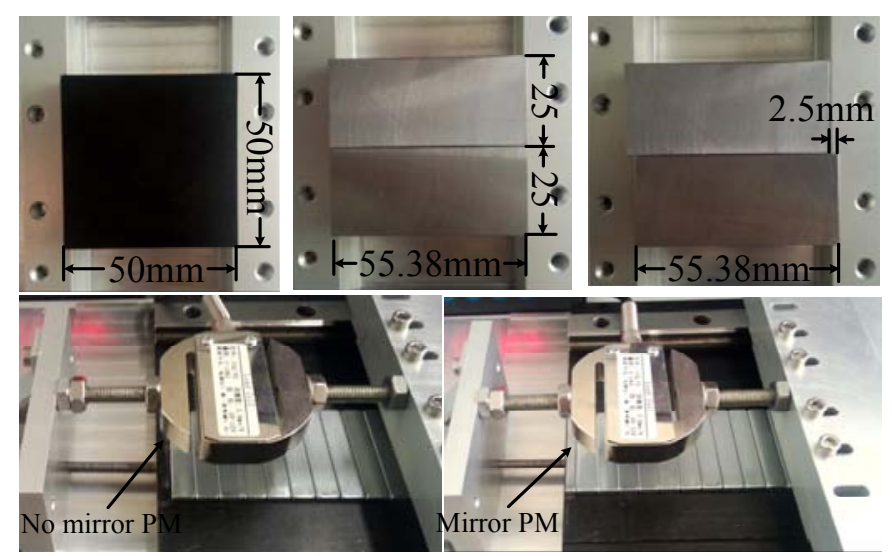

Fig. 22. The slotless LPMSM prototype.

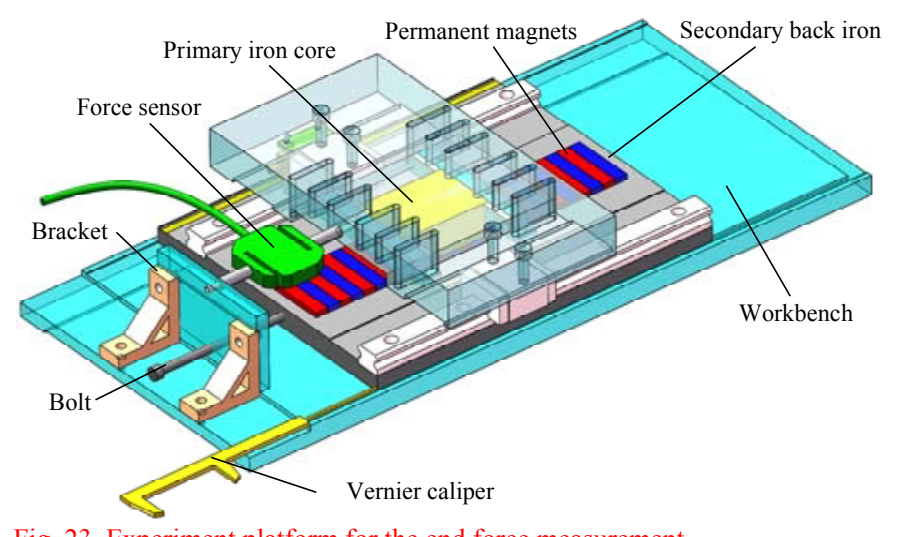

Fig. 23. Experiment platform for the end force measurement. 


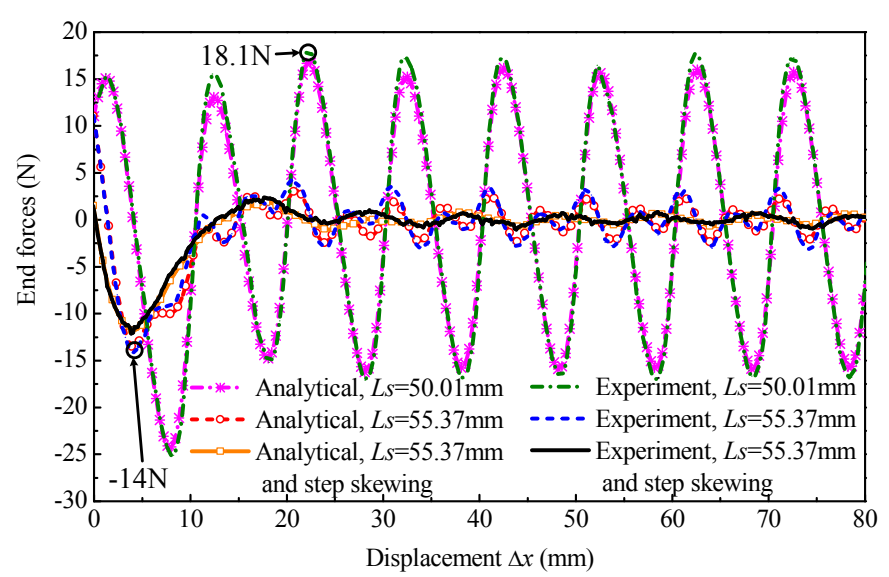

(a)

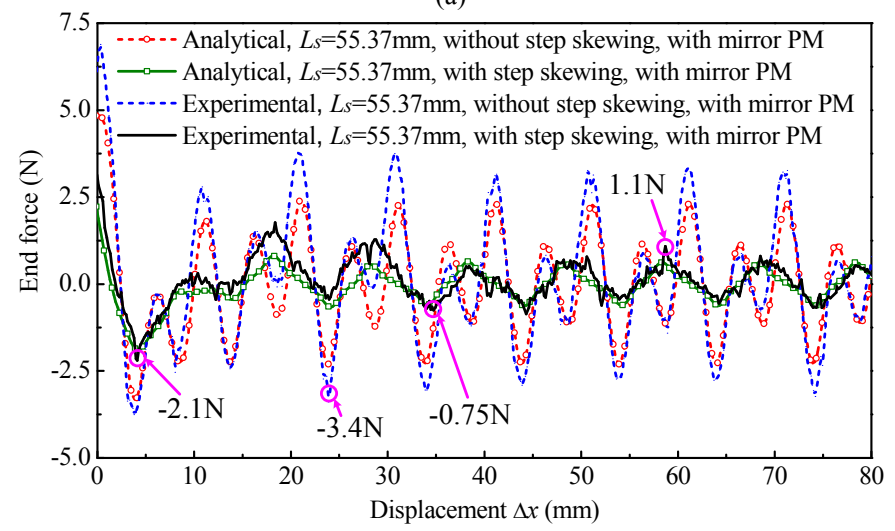

(b)

Fig. 24. Comparison of tangential thrust obtained by FEM, analytical and experiment. (a) Without mirror PM; (b) With added mirror PM.

TABLE X

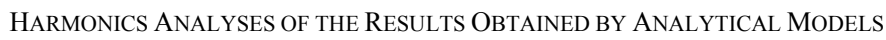
AND EXPERIMENTAL TESTS

\begin{tabular}{|c|c|c|c|c|c|c|}
\hline Harmonics & DC & 1 & 2 & 3 & 4 & 5 \\
\hline Data 1 & 0 & 15.75 & 0.93 & 0.39 & 0.47 & 0.40 \\
\hline Data 2 & 0.86 & 16.86 & 2.92 & 0.66 & 0.37 & 0.13 \\
\hline Data 3 & 0.04 & 0.68 & 1.67 & 0.12 & 0.01 & 0.04 \\
\hline Data 4 & 0.02 & 1.66 & 1.86 & 0.03 & 0.09 & 0.06 \\
\hline Data 5 & 0.05 & 0.48 & 0.11 & 0.06 & 0.05 & 0.01 \\
\hline Data 6 & 0.08 & 0.57 & 0.13 & 0.02 & 0.04 & 0.02 \\
\hline
\end{tabular}

*Data 1 - Analytical, $L_{s}=50 \mathrm{~mm}$; Data 2 - Experimental, $L_{s}=50 \mathrm{~mm}$; Data 3 Analytical, $L_{s}=55.38 \mathrm{~mm}$; Data $4-$ Experimental, $L_{s}=55.38 \mathrm{~mm}$; Data 5 Analytical, $L_{s}=55.38 \mathrm{~mm}$ and step-skewed auxiliary irons; Data 6 Experimental, $L_{s}=55.38 \mathrm{~mm}$ and step-skewed auxiliary irons.

\section{CONCLUSION}

In this paper, the accurate calculation of the left and right end forces in LPMSMs is firstly investigated according to an analytical model and the Maxwell stress sensor. Secondly, the optimal length of the primary is obtained by the spectrum analyses of the left/right end forces and a two-step iteration, and the fundamental is almost completely eliminated. Then, the second harmonic is eliminated by adopting step-skewed auxiliary irons. Thirdly, by adding mirror PMs to the ends of the secondary back-iron, the secondary end effect is also reduced. Finally, an LPMSM prototype is manufactured and experiments are conducted. The effectiveness of end force analysis and minimization is validated by the experiments.

\section{REFERENCES}

[1] W. X. Zhao, J. Q. Zheng, J. B. Wang, G. H. Liu, J. X. Zhao, and Z. Y. Fang, "Design and analysis of a linear permanent-magnet vernier machine with improved force density," IEEE Trans. Ind. Electron., vol. 63, no. 4, pp. 2072-2082, Apr. 2016.

[2] B. J. Kang and C. M. Liaw, "A robust hysteresis current-controlled PWM inverter for linear PMSM driven magnetic suspended positioning system," IEEE Trans. Ind. Electron., vol. 48, no. 5, pp. 965-967, Oct. 2001.

[3] Z. Z. Liu, F. L. Luo, and M. A. Rahman, "Robust and precision motion control system of linear-motor direct drive for high-speed X-Y table positioning mechanism," IEEE Trans. Ind. Electron., vol. 52, no. 5, pp. 1357-1363, Oct. 2005.

[4] M. Y. Chen and J. S. Lu, "High-precision motion control for a linear permanent magnet iron core synchronous motor drive in position platform," IEEE Trans. Ind. Inform., vol. 10, no. 1, pp. 99-108, Feb. 2014.

[5] M. Inoue and K. Sato, "An approach to a suitable stator length for minimizing the detent force of permanent magnet linear synchronous motors," IEEE Trans. Magn., vol. 36, no. 4, pp. 1890-1893, Jul. 2000.

[6] M. Y. Wang, L. Y. Li, and D. H. Pan, "Detent force compensation for PMLSM systems based on structural design and control method combination," IEEE Trans. Ind. Electron., vol. 62, no. 11, pp. 6845-6854, Nov. 2015.

[7] L. Z. Zeng, X. D. Chen, X. Q. Li, W. Jiang, and X. Luo, "A thrust force analysis method for permanent magnet linear motor using Schwarz-Christoffel mapping and considering slotting effect, end effect, and magnet shape," IEEE Trans. Magn., vol. 51, no. 9, art. 8107609, Sep. 2015.

[8] F. Cupertino, P. Giangrande, G. Pellegrino, and L. Salvatore, "End effects in linear tubular motors and compensated position sensorless control based on pulsating voltage injection," IEEE Trans. Ind. Electron., vol. 58, no. 2, pp. 494-502, Feb. 2011.

[9] I. S. Jung, J. Hur, and D. S. Hyun, "Performance analysis of skewed PM linear synchronous motor according to various design parameters," IEEE Trans. Magn., vol. 37, no. 5, pp. 3653-3657, Sep. 2001.

[10] J. Faiz, M. Ebrahimi-Salari, and G. Shahgholian, "Reduction of cogging force in linear permanent-magnet generators," IEEE Trans. Magn., vol. 46, no. 1, pp. 135-140, Jan. 2010.

[11] T. Liu, S. D. Huang, J. Gao, and K. Y. Lu, "Cogging force reduction by slot-opening shift for permanent magnet machine," IEEE Trans. Magn., vol. 49, no. 7, pp. 4028-4031, Jul. 2013.

[12] G. J. Li, B. Ren, Z. Q. Zhu, Y. X. Li, and J. Ma, "Cogging torque mitigation of modular permanent magnet machines," IEEE Trans. Magn., vol. 52, no. 1, art. 8100210, Jan. 2016.

[13] A. M. EL-Refaie, "Fractional-slot concentrated-windings synchronous permanent magnet machines: opportunities and challenges," IEEE Trans. Ind. Electron., vol. 57, no. 1, pp. 107-121, Jan. 2010.

[14] Z. Q. Zhu, Z. P. Xia, D. Howe, and P. H. Mellor, "Reduction of cogging force in slotless linear permanent magnet motors," IEE Proc. - Electr. Power Appl., vol. 144, no. 4, pp. 277-282, Jul. 1997.

[15] C. Y. Liu, H. T. Yu, M. Q. Hu, Q. Liu, and S. G. Zhou, "Detent force reduction in permanent magnet tubular linear generator for direct-driver wave energy conversion," IEEE Trans. Magn., vol. 49, no. 5, pp. 1913-1916, May 2013.

[16] Y. S. Kwon and W. J. Kim, "Detent-force minimization of double-sided interior permanent-magnet flat linear brushless motor," IEEE Trans. Magn., vol. 52, no. 4, art. 8201609, Apr. 2016.

[17] C. C. Hwang, P. L. Li, and C. T. Liu, "Optimal design of a permanent magnet linear synchronous motor with low cogging force," IEEE Trans. Magn., vol. 48, no.2, pp. 1039-1042, Feb. 2012.

[18] N. Baataro, H. S. Yoon, M. T. Pham, P. S. Shin, and C. S. Koh, "Shape optimal design of a 9-pole 10-slot PMLSM for detent force reduction using adaptive response surface method," IEEE Trans. Magn., vol. 45, no. 10, pp. 4562-4565, Oct. 2009.

[19] Y. W. Zhu, D. H. Koo, and T. H. Cho, "Detent force minimization of permanent magnet linear synchronous motor by means of two different methods," IEEE Trans. Magn., vol. 44, no. 11, pp. 4345-4348, Nov. 2008.

[20] N. M. Kimoulakis, A. G. Kladas, and J. A. Tegopoulos, "Cogging force minimization in a coupled permanent magnet linear generator for sea wave energy extraction applications," IEEE Trans. Magn., vol. 45, no. 3, pp. 1246-1249, Mar. 2009.

[21] H. Z. Hu, J. Zhao, X. D. Liu, Z. Chen, Z. X. Gu, and Y. Sui, "Research on the torque ripple and scanning system of an arc-structure PMSM used for scanning system," IEEE Trans. Magn., vol. 50, no. 11, art. 8105504, Nov. 2014. 
[22] H. Z. Hu, J. Zhao, X. D. Liu, and Y. G. Guo, "Magnetic field and force calculation in linear permanent-magnet synchronous machines accounting for longitudinal end effect," IEEE Trans. Ind. Electron., vol. 63, no. 12, pp. 7632-7643, Dec. 2016. 